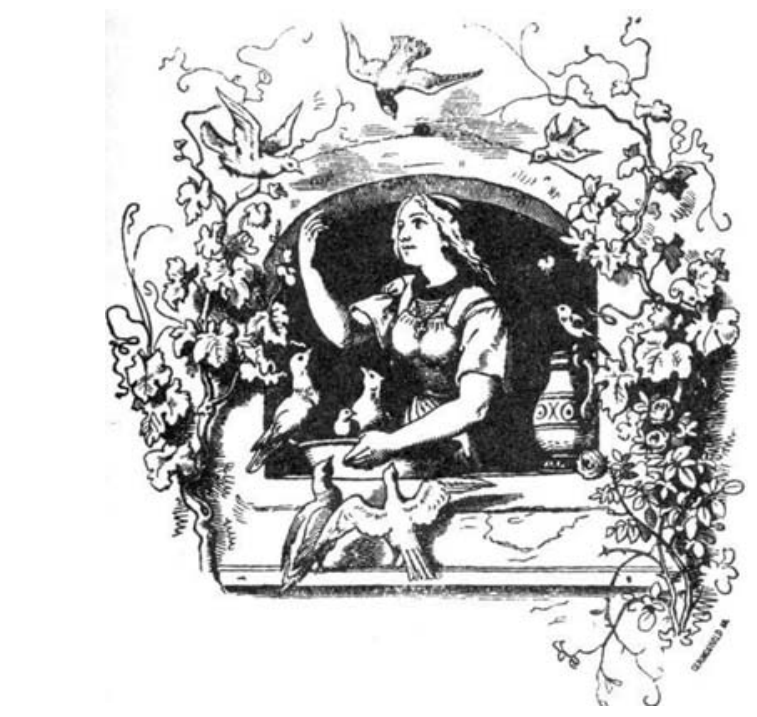

Aschenputtel bei der Durchführung selektiver Aufgaben, die ihr von einer bösen Frau auferlegt wurden. Gezeichnet von Ludwig Richter

\title{
Die schlechten ins Kröpfchen?
}

\section{von Alfred Schreiber}

Im ersten Band von Mathematik als pädagogische Aufgabe ${ }^{1}$ bemerkt Hans Freudenthal: „Mathematik ein zuverlässiges Selektionsmittel ...? Es ist nun leider so, daß dies das Einzige ist, was über den Allgemeinwert der Mathematik wirklich mit großer Sicherheit feststeht." (S. 82) - Eine leicht selbstquälerische Bemerkung. Freudenthal beschreibt die „Selektion in intellektuellen Funktionen" durch das Schulfach Mathematik denn auch als einen trennscharfen, häufig peinvollen Vorgang. In der Tat, es lässt sich vergleichsweise leicht und zuverlässig erkennen, wenn jemand den mathematischen Stoff nicht verstanden hat oder den Leistungsanforderungen des Fachs nicht gewachsen ist. In dem Fall sitzt der Stachel schon einmal tief und sorgt - verständlicherweise - lebenslang für Ressentiments. Deren noch gemäßigter Ausdruck ist die mittlerweile geläufige Kokettierphrase: In $M a$ the war ich immer schlecht...

Das Thema Selektion ist ein vermintes Feld, und auch der sonst so souveräne Argumentierer Freudenthal vermag es nur in einem dialektischen Eiertanz zu überqueren. Einerseits ist es eine schlichte Tatsache, dass Mathematik als Selektionsmittel taugt und geschätzt wird. Andererseits - und jetzt ist es gut, sich nicht allein zu wissen - wollen wir (!) dies bedauern, jedenfalls im Prinzip, nämlich in ganz bestimmten Rollen: „Wir wehren uns dagegen als Erzieher, die sich weigern, die Selektion als Ziel des Erziehungsprozesses anzuerkennen, und als Mathematiker, die meinen, daß die Mathematik dazu zu gut sei." Letzteres werden natürlich auch andere Fächer mit einigem Recht für sich in Anspruch nehmen wollen. Dass Selektion kein sinnvolles Ziel von Bildung ist, scheint mir zudem ein selbstgängiges Argument. Es stützt wohl kaum eine weitgehende oder generelle Ablehnung von Prüfungen, zu der sich etwa Giuseppe Peano bekannt haben soll, dessen „tolerance of human weakness“ sein Biograph H. C. Kennedy ausdrücklich rühmt.

„Doch wird noch selektiert" - beklagt sich gleichwohl Freudenthal. Und vielleicht hat das den von Außenstehenden üblicherweise vorgetragenen (sprich: vorgeschobenen) Zweck, dass so nicht „alles Arzt, Apotheker oder Rechtsanwalt werden" kann - lauter angesehene Berufe also, die Zugehörigkeit zum Club der Besserverdienenden versprechen. Ein wenig gereizt wirft Freudenthal ein: „Eine Lotterie wäre vielleicht zweckmäßiger, aber wer akzeptiert das?". Ja, ganz recht. Ebensogut könnte man fragen, wer sich gerne einem ungeprüften Piloten anvertrauen oder über Brücken fahren und in Häusern wohnen möchte, die von nicht-selektierten (oder per Los bestimmten) Ingenieuren und Architekten gebaut wurden. Und kaum einer - um zu harmloseren Dingen zurückzukehren - besucht gerne ein Konzert mit schlecht spielenden Musikern. Wenig später fällt dann doch der Satz: „Prüfen ist eine sinnvolle Tätigkeit." Allerdings führt Freudenthal nicht jene eben erwähnten Ärzte, Ingenieure und Piloten ins Feld, sondern

1 Stuttgart: Klett Verlag, 1973 
macht geltend, der Unterrichtende könne auf diese Weise „den Erfolg des Lehrprozesses nachprüfen“; außerdem hätten der Unterrichtete und die „Außenwelt“ ein Recht darauf $\mathrm{zu}$ wissen, ob etwas gelernt worden sei. Punktuelle Beurteilungen seien zu vermeiden (eigentlich selbstverständlich) sowie „Unterricht und Prüfung nicht voneinander zu trennen" - letzteres weniger selbstverständlich und eine, wie ich finde, nicht unbedingt einladende Perspektive. Der Lehrer soll „der Prüfung die Schärfe des Selektionsmittels nehmen" und den "goldenen Mittenweg zwischen Milde und Strenge" suchen. "Ich kann bei dem Gegenstand nicht zu lange verweilen. Die Wirklichkeit ist nun einmal die ..." (S. 83 f.). Man spürt, wie sehr das Thema dem Autor auf die Nerven geht. Er belässt es schließlich - nicht ungeschickt - bei einem pädagogisch abgefederten Kompromiss, der sich gerade noch mit den Anforderungen der gesellschaftlichen Realität verträgt. Man ist ja kein Unmensch. Und das Leben ist nun mal voller Risiken.

Das weiß auch die Pädagogik und hält ihre schützende Hand zunächst einmal vor diejenigen, die von der Selektion in der ersten Reihe betroffen sind, also z. B. die noch studierenden künftigen Lehrerinnen und Lehrer. Die in zweiter Reihe betroffenen Kinder geraten dabei leicht aus dem Blickfeld. Aber verdienen sie nicht im selben Maße Schutz und Fürsorge wie die Passagiere eines Flugzeugs, die jederzeit davon ausgehen können (müssen), dass das technische System (samt der darin arbeitenden Personen), dem sie sich anvertrauen, geprüft ist und hohen, wenn nicht höchsten Sicherheitsanforderungen genügt? Hat nicht die Gesellschaft das Recht auf eine vergleichbare Sorgfalt, wenn es um das Personal geht, das sich demnächst mit Geist und Seele Heranwachsender beschäftigt?

Ein Beispiel in der Tradition pädagogischer Hochschulen sind die fachdidaktischen Schulpraktika. Sie bilden - neben einer soliden Ausbildung im Fach gewiss einen substanziellen Beitrag zu der Ausübung der eben angemahnten Sorgfalt. Wer sich auf den Lehrberuf vorbereitet, braucht Gelegenheiten, sich in die neue Rolle einzuüben, und Handlungsfelder, um didaktische Entwürfe seines Fachs an der Realität abzuarbeiten und gegebenenfalls zu revidieren. Es bleibt zu fragen, in welchem Maße Studierende dabei auch eine falsche Berufswahl von sich aus revidieren. Die Erfolgsquoten, die ich aus eigenen Erfahrungen kenne, könnten Außenstehenden den frappierenden Eindruck vermitteln, es gebe hier so gut wie keine falschen Entscheidungen. Nicht nur Peano oder Freudenthal - so vermute ich einmal - hat dieses Thema nervös gemacht.

\author{
Adresse des Autors \\ Prof. Dr. Alfred Schreiber \\ Institut für Mathematik und ihre Didaktik \\ Universität Flensburg \\ Auf dem Campus 1 \\ 24943 Flensburg \\ alfred.schreiber@uni-flensburg.de
}

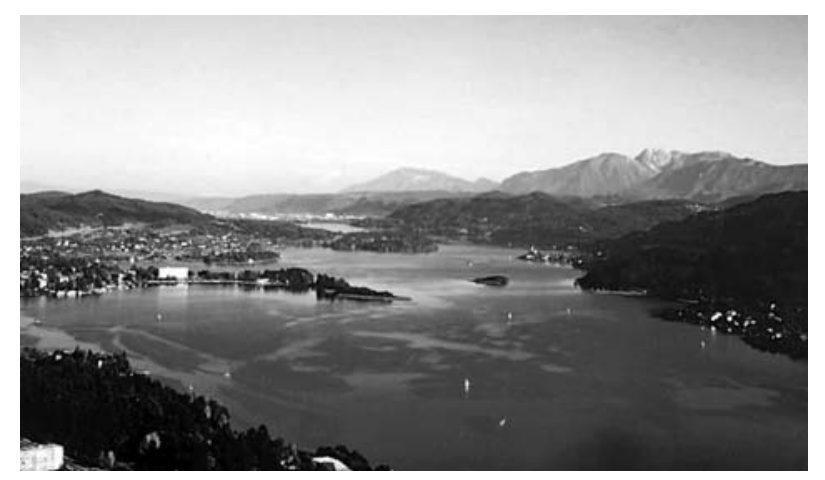

\section{Internationaler Mathematikerkongress der ÖMG und Jahrestagung 2005 der DMV} Klagenfurt 18.-23. September 2005

Die örtliche Tagungsleitung möchte nochmals alle an Mathematik Interessierte herzlich einladen, den 16. Internationalen Mathematikerkongress der Österreichischen Mathematischen Gesellschaft, zugleich die Jahrestagung 2005 der Deutschen MathematikerVereinigung vom 18.-23. September 2005 an der
Alpen-Adria-Universität Klagenfurt zu besuchen. Ab nun ist auch die Registrierung über die Homepage http://oemg2005. uni-klu.ac.at möglich. Weiteres entnehmen Sie bitte dieser Seite.

Die örtliche Tagungsleitung 